\title{
Ambient Assisted Living Technology: Comparative perspectives of users and caregivers
}

Conference Paper · October 2013

DOI: 10.1109/HealthCom.2013.6720635

READS

10

7 authors, including:

Gabriela Trevisan

ESEPF/CIPAF e CIEC/Uni. do Minho

13 PUBLICATIONS 4 CITATIONS

SEE PROFILE

José Ferreira-Alves

University of Minho, Braga

34 PUBLICATIONS 52 CITATIONS

SEE PROFILE
Liliana Ferreira

Associação Fraunhofer Portugal Research

38 PUBLICATIONS 65 CITATIONS

SEE PROFILE 


\title{
Ambient Assisted Living Technology: Comparative perspectives of users and caregivers
}

\author{
David Cunha*, GabrielaTrevisan*, Florbela Samagaio*, \\ Liliana Ferreira $^{\dagger}$, Filipe Sousa ${ }^{\dagger}$, \\ Jose Ferreira-Alves ${ }^{\ddagger}$, and Ricardo Simoes ${ }^{\S}$ \\ * Dept Social Education Paula Frassinetti School of Education Rua Gil Vicente, 138 4000-225 Porto Portugal \\ $\dagger$ Fraunhofer Portugal AICOS, Rua Alfredo Allen, 455 4200-135 Porto Portugal \\ ${ }^{\ddagger}$ School of Psychology University of Minho 4710-057 Braga Portugal \\ $\S$ Institute for Polymers and Composites IPC/I3N, University of Minho, Guimarães; \\ Life and Health Sciences Research Institute (ICVS), University of Minho, Braga; \\ School of Technology, Polytechnic Institute of Cavado and Ave, 4750-810 Barcelos, Portugal
}

\begin{abstract}
Developed societies are currently facing severe demographic changes: the world population is ageing at an unprecedented rate. This demographic trend will be also followed by an increase of people with physical limitations. New challenges are being raised to the traditional health care systems, not only in Portugal, but also in all other European states. There is an urgent need to find solutions that allow extending the time people can live in their preferred environment by increasing their autonomy, self-confidence and mobility. AAL4ALL is a project currently being developed in cooperation with 34 Portuguese interdisciplinary partners, from industry to academia, $R \& D$ and social disciplines, which employs a novel conceptual approach through the development of an ecosystem of products and services for Ambient Assisted Living (AAL) associated to a business model and validated through large scale trial. This paper presents a comparative perspective of the needs and attitudes towards technology of the AAL users and caregivers identified in the analysis of a set of three different surveys: a users survey targeted at the Portuguese seniors and pre-seniors; an informal caregivers survey targeted at the family, friends and neighbours who provide care without any financial compensation; and a formal caregivers survey targeted at physicians, nurses, psychologists, social workers, and direct-care workers providing care to elders. The first results indicate that AAL solutions must be affordable, user friendly and have a true perceived benefit to their users.
\end{abstract}

\section{INTRODUCTION}

AAL solutions aim at supporting people with special needs through technology, so physically impaired and elderly people can maintain an independent living in their own homes. AAL products have the potential to improve autonomy and quality of life, but also to reduce associated costs to health services of elderly people.

AAL technology also tries to enhance quality of life (at a physical, mental and social level) to all kinds of users throughout different stages in life. AAL can support people with specific needs through intelligent interaction with surrounding environments mediated by devices. The AAL concept represents a real paradigmatic change in person-computer interaction. Going from a personal device/computer to a multiplicity of devices capable of processing and computing data from the environment is a new paradigm creating new challenges to peoples interaction with technology.
In Portugal, and according to Portuguese Statistics, a continuous demographic ageing can be observed as a result of different tendencies of higher life expectancy, life conditions and lower natality rates [1]. The proportion of elderly people represented, in 1960, 8\% of the population, whereas in 2009 it more than doubled to $17,9 \%$ [1], [2]. Alongside these increases in ageing rates we can also observe an increase of dependency levels (that is, the relation between elderly people and active population in Portugal) and of life expectancy levels that increase complexity of ageing issues.

AAL4ALL is a project currently being developed in cooperation with 34 Portuguese interdisciplinary partners, ranging from industry to academia, R\&D and social disciplines. The main goal of the project is the mobilization of an industrial ecosystem of products and services in the scope of AAL and is specially focused on the definition of specific standards. Given this goal, the project started by specifying the requirements of senior and pre-senior users and their caregivers by using dedicated surveys. These data allowed to understand how information and communication technologies (ICT) are already part of the daily activities of the target users and of their caregivers and to define new markets for care products and services. The developed surveys are focused on the identification and characterization of the needs of the final users in terms of AAL solutions and their technology acceptance. This procedure will allow the development of a user-centric model, capable of answering the needs of the users while ensuring an optimal integral assistance, improving the quality of life and the wellbeing of individuals and their caregivers [3].

This paper starts by introducing in Section II the background knowledge that supports the AAL4ALL dedicated surveys. Section III presents the research methodology adopted in the creation of each group of the questionnaires: it introduces sampling methods and procedures used to conduct the survey. A comparison on the perspectives and priorities identified on each of the surveys is presented and discussed in Section IV. A discussion of the main conclusions supported by these results is presented in section V, while section VI concludes the paper by resuming the results of the described analysis. 


\section{BACKGROUND AND RELATED STUDIES}

Caregiving is a task of vital importance when analyzing the living situations of dependent and/or older people. As age evolves and capacities start to decline, most of older adults will need support. This support is usually carried out by family members who, alongside with a more or less present formal support, ensure that the basic needs of the care receiver are fulfilled. Dependence generally translates into lower personal autonomy, which leads to a growing need to support the individual in their environment and their interactions with it. The term caregiving associated to informal care is usually defined as "relation networks between family members, friends and neighbors" [4] that provide care and supervision to a dependent person in a non paid way for them to achieve an admissible level of satisfaction in their needs. This care can occur at an instrumental, personal, affective, cognitive, and economic level and varies in intensity and time according to the needs [5]. The caregiving network size is often viewed as an indication of the range and depth of resources that can be leveraged for assistance. A large network with additional helpers may alleviate the burden on a primary caregiver. A larger network has also been shown to contribute to the reduction of the hours of help given per day, to the continuation of caregiving over a longer period of time, and to a greater variety of tasks that are performed to support frail older people. Caregiving is, as mentioned previously, related with the daily life and instrumental activities and with providing psychological and affective support. If we recognize the unsustainable pressures on the health and care systems that help older, sick or disabled people, so we have to recognize the unsustainable pressures on the families who so often make up the shortfall in their services [6].

We all care or will have to care at some point in our lives, most often for a family member. Technology has a vital part to play in supporting families to care, in supporting health and care services to enable them to do so without penalty, and in supporting employers to help people combine work and care [6].

Technology solutions have to be the right solutions, genuinely offering choice, independence and control to individuals and families, and taking account of their lifestyle needs. Regarding formal caregivers, it is important to understand what role they currently play in health care of elders. The social network near dependent persons, especially immediate family, is a fundamental source of help in providing the care tasks. However, on many occasions it is necessary to recruit additional help or to get more specialized services offered by professionals from different fields, which can attend to different needs. These needs may include health care, social care and daily tasks, depending on the specificity of the individuals.

Formal caregivers can be divided into two categories:

1) professionals include nurses, physicians, therapists, and social workers; and

2) direct-care workers include home health aides, homemakers, companions, and patient care attendants.

The formal care is thus the provision of care normally carried out by suitably qualified professionals (doctors, nurses, psychologists, social workers, educators, among others) and is therefore designated as formal. Formal caregivers are paid professionals, who through formal training, education and experience, bring specialized knowledge and expertise to the care receiver. There is a specific preparation for the performance of this role, being integrated within a professional activity.

In most industrialized countries, the need for home technologies to assist patients has been increasing whether for short periods of care or for chronic conditions. Two social phenomena seem to justify the increase on high-tech home care: on the one hand, asking the patients to become active users of health technologies, and on the other hand, the idea that treatments could be made in different locations aside from just hospitals.

These technologies also ultimately intend to provide end users with more autonomy even if assisted by professional and formal caregivers, as it often happens with older people. If autonomy is understood here as a way for older people to manage their life in their preferred environment preferably their home reinforcing mobility and quality of life and improving access to technologies, then the role of formal caregivers in helping these transitions to technology use could be very important. These technologies can then facilitate care giving professionals in their daily tasks but also assure that users have resources to face their main difficulties.

\section{Methodology}

The AAL4ALL surveys were developed through an iterative process addressed by a subgroup of industrial and academic partners of the AAL4ALL project. Each questionnaire was created after the initial segmentation of the target group and the definition of the main research questions to be addressed by these instruments. They covered all Portuguese regions, and both rural and urban settings. The estimation of the sampling size of each survey was conducted before the application of the survey in order to achieve a margin error of $3 \%$ at $95 \%$ confidence. However, the use on each survey of a non-probabilistic sampling method, namely, convenience sampling and, consequently, the impossibility to determine accurately the probability of the subject selection, does not allow the accurate estimation of the sampling error. The use of a non-probabilistic sampling method is justified with the unavailability of the sampling frame of the target groups and the need to ensure that samples can be monitored for some time.

\section{A. Users Survey}

The users survey is composed of 8 main sections, each focusing on different aspects of the 50+ population life: sociodemographics, residence characteristics and social spaces, health measures, functionality, security, social activities, services, and attitudes towards technology. The data were collected from the universe of Portuguese citizens aged 50 or more, characterized, at the date of the questionnaire, by one of the following settings:

- $50+$ citizens attending residential facilities;

- $\quad 50+$ citizens attending day care centres; 
- $50+$ citizens living in their own home with some kind of integration in the community (e.g. senior universities, associations, citizen public services);

- $\quad$ Other citizens aged 50+.

In order to facilitate adequately communication of the purpose of the survey and to ensure the standardization of the filling procedure, it was interviewer-mediated. The interviewer was responsible for explaining the study to the respondent, the importance of their participation and register their answers. All data were collected simultaneously in the different regions of Portugal between July and September 2011. In each region, one or more local coordinators supervised the sample selection, the recruiting and the interviewers training. The respondents were selected mainly from residential facilities and day care centres but also in senior universities, associations and citizen public services.

\section{B. Informal Caretakers Survey}

The informal caretakers survey is composed of 5 main sections, each focusing on different aspects of the caregiving, namely: socio-demographics of the informal caregiver, data on the provided care, socio-demographic and clinical data of the care receiver, main services used by the caregiver, and attitude towards new technology. The sample was selected from the universe of Portuguese informal caregivers, more precisely family, friends and neighbors who provided care without a formal character (or financial compensation) independently of their age, gender, residence area or any other socio-demographic factor. A set of guidelines was created and distributed to all the partners containing detailed instructions on how to present each of the survey questions to the respondent. The final version of the survey, a paper instrument produced with a TeleForm application and an online version, was shared with all project participants on December 2011. All data were collected simultaneously in different regions of Portugal between December and March, 2012. In each region, one or more local coordinators supervised the sample selection.

\section{Formal Caretakers Survey}

The third survey developed within the AAL4ALL project was targeted at the formal caregivers of the targeted population. The main objective of this survey was to understand how ICT were already part of the daily activities of these target users the formal caregivers. The initial segmentation of the target group allowed identifying four main groups of formal caregivers: physicians and nurses, psychologists, social workers, and direct care workers. One questionnaire was developed for each of the identified groups and each questionnaire was comprised of 3 main sections, each focusing on different aspects of the caregiving, namely: socio-demographic characterization of the formal caregiver, attitude toward new technology, and care receivers characterization. The formal caregivers sample was selected from the universe of Portuguese formal caregivers, independently of their age, gender, residence area or any other socio-demographic factor. The final version of the survey, paper instrument produced with a TeleForm application, and online versions were shared with all project participants in the end of March 2012, together with detailed instructions on how to present each of the survey questions to the respondent.
All data were collected simultaneously in different regions of Portugal between April and June 2012. In each region, one or more local coordinators supervised the sample selection.

\section{RESULTS}

\section{A. Socio Demographics}

The sample of the first survey targeted at users is composed by 788 women and 370 men in the age range of 50 to 98 years old. The majority of participants are married (502) and widowed (457), with a lower presence of single (116) or divorced (72) subjects. Most of the respondents have no more than four years of school education $(69.1 \%)$ and the large majority $(74.3 \%)$ is retired and does not live alone $(53.7 \%)$. About $54.2 \%$ of the respondents earn less than 734 per month (Figure 1). Also, the analysis of the survey data allowed determining that $50 \%$ of the respondents live in an independent house while $25 \%$ of the subjects live in specially designed housing for older adults, with permanent care. The results indicate, thus, that most of the respondents are still living with family or in an autonomous way.

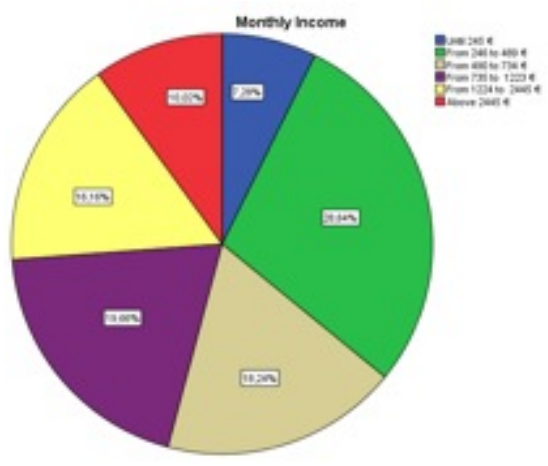

Fig. 1. Distribution of the monthly income of the users study sample.

Regarding the survey on informal caregivers, we were able to collect data on the individual caregiving circumstances of 268 Portuguese citizens aged 20 to 92 years old in 17 regions of the mainland country. The number of women (188) in our sample is more than twice the number of men (71). The large majority $(40.3 \%)$ of our participants still have a paid occupation while $24.6 \%$ are retired. The most frequent relationship of the caregiver with the care receiver is the parent-child (father/mother) relationship (33.6\%), followed by the son/daughter $(19.0 \%)$, husband/wife/partner $(13.8 \%)$ and grandson/granddaughter $(10.8 \%)$ relationships. About $59 \%$ of the respondents report giving care every day (Figure 2).

By conducting the survey on formal caregivers we were able to collect data from 502 respondents, from which 217 are physicians and nurses, 34 psychologists, 95 social workers and 156 direct care workers. The large majority $(95.5 \%$ in the case of direct care workers) are women.

\section{B. Attitudes toward Technology}

The analysis of the attitudes of the subjects towards technology is one of the most relevant and more significant components of the AAL4ALL surveys. Results indicate that the majority of the subjects that responded to the users survey 


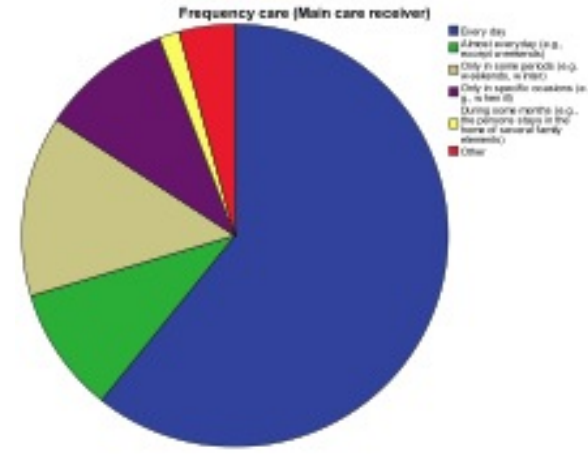

Fig. 2. Informal caregivers frequency of care distribution.

have access to cell phones, whilst one third of the respondents do not own such a device. Most of the respondents have access to Internet at home; however, approximately, two thirds of the inquired individuals have never accessed the Internet. An analysis more focused on the use of AAL technologies reveal that one third of the respondents has never used this type of solutions. The majority, however, report some experience with AAL technologies independently of their type. Difficulties in the use of this type of technology are reported by most of the subjects and half of the sample population refers the availability to invest in the acquisition of AAL technologies. Although approximately $50 \%$ of the subjects have income below 734 per month, a large majority of the subjects indicated that they are available to pay for AAL technologies that may increase their quality of life.

Data obtained from informal caregivers indicate that $49 \%$ of the respondents already use technology to support their caregiving activities. Most of them agree that technology is useful for their work as caregivers $(68.2 \%)$ and is able to improve their well-being (64.6\%). Regarding internet, the informal caregivers indicate that they mostly use it to read news $(15.2 \%)$, search for products and services (11.9\%) maintain contact with family and friends (11.4\%) and access services $(10.7 \%)$. Also, most of the informal caregivers have confidence on the information available online $(58.2 \%)$.

When analyzing the data from the formal caregivers surveys, we were mostly interested in understanding how technology is already being used in their daily professional activities and which are the perceived benefits and barriers of technology usage. The first group of formal caregivers analyzed - physicians and nurses, indicate that the main ICT benefits are related to the facilitation of the integration of the patient information $(73.7 \%)$ and the increase of collaboration among the multidisciplinary team $(81.6 \%)$. Psychologists report perceived benefits that are also related with the easier integration of the patient information $(73.5 \%)$, but also identify the promotion of a better clinical and psychosocial management of patients $(55.9 \%)$ and the improved access to psychology related services $(50 \%)$ as relevant benefits. Social workers identify as ICT utilization benefits the better communication and collaboration with the multidisciplinary team $(82.1 \%)$, the easier access to the information made available by the social security services $(66.3 \%)$, and an improvement on counseling clients $(53.7 \%)$. Direct-care workers refer to the higher client satisfaction with the higher focus on illness prevention and health promotion $(80.8 \%)$ provided by ICT utilization. On the other side, physicians and nurses identify as barriers to the utilization if ICT the lack of available equipment (48.8\%), and the overhead effort required to use ICT (46.1\%). Psychologists showed an high concern with the security and confidentiality of the user data $(47.1 \%)$ while most of the social workers referred to the ICT associated costs and the lack of public incentives to ICT acquisition as some of the main barriers related with ICT utilization ( $42.1 \%$ and $33.7 \%$, respectively). Finally, the perceived barriers of ICT utilization for direct care workers are mostly related with the related overhead $(54.5 \%)$, the lack of equipment $(52.6 \%)$, and the low level of expertise in ICT utilization (51.9\%) among this class of workers.

\section{Discussion}

Introducing new technologies in older people lives aims to favour interpersonal communication, and will affect the current way of communication for this group of people [7]. New technologies allow older adults to integrate in a new social support group by bringing them closer to youngsters, therefore creating a rich environment for sensorial stimulation. It is also believed that new technologies promote principles of solidarity between generations, increasing, for instance, volunteer actions and transmission of personal and professional experiences [8]. Life expectancy has increased leading to a higher number of older adults dependent on help from caregivers for everyday life tasks that frequently depend on informal caregivers to provide it. Given the analysis and data provided, this is a key group since they are often the main decision makers on using/acquiring AAL services and products. Independence and autonomy are two important levels when considering AAL services and products, since it is possible to design them for people with different needs. Hence, the idea that AAL would only interest those not fully independent can be challenged since AAL can also be seen as helpful to improve quality of life in fully independent individuals. Home services by specialised companies, whether public or private, are growing in Portugal (particularly in the private sector). In the same sense, a concern on developing differentiated services to this population, could justify AAL investments in order to help older people to remain in their home environment as long as possible. On conceiving AAL products and services this information is particularly relevant since it must be considered to be able to build user friendly technologies [9]. Pertinence of these services is even larger if we consider that most of the respondents are still working. It should also be considered when creating AAL technologies that prices should conform to realistic possibilities by older adults and caregivers, unless public social policies are expected to assume part of the cost. ICT can have a very important role in care giving contexts essentially as an effective way to inform and improve knowledge from care givers on the conditions of specific diseases, on ageing and on handling handicapped conditions. They can also help caregivers find specific support groups and networks in their own communities, including to share their own difficulties during difficult periods of care giving. They could also be seen as effective tools to assess risk in health conditions and of promoting health. Different research has shown how computers are being used in therapeutic interventions to help caregivers in anger management, reducing depression symptoms [9]. These 
results in different studies are still recent and try to explore the effects of technological interventions guided to help caregivers, their benefits, and users responses. It is also necessary to consider the fact that most caregivers will eventually end up as recipients of care themselves, either due to their advanced age or to the burnout of the care giving activities [10]-[12]. In this sense, both the elders currently receiving care and also their caregivers can benefit from AAL solutions and the promotion of well-being strategies.

\section{CONCLUSION}

With the changing demographics, Ambient Assisted Living (AAL) technologies can be an important role in improving the quality of life, the quality of services, and the cost effectiveness of health care. It is important, however, to understand the perspectives of all the different players involved, namely the care receivers, informal care givers, and formal care givers. The information extracted from surveys to each of these groups (conducted on a relatively significant sample), indicate some of the most pertinent aspects they consider as benefits or obstacles to the use of AAL solutions. Overall, the conducted studies allow to identify several perspectives of the users and caregivers on the technological aspects of AAL: the study focused on the users indicate that subjects report some kind of difficulty in using AAL solutions, but also willingness and confidence to learn how to use it. Finally, both caregivers studies results indicate that AAL solutions must be affordable, user friendly and have a true perceived benefit by their users in caregiving tasks. The results of these surveys will be used to create recommendations directed to the other working teams of the AAL4ALL project in order to allow the development of a validated prototype and support the defined business models.

\section{ACKNOWLEDGMENT}

Project AAL4ALL, co-financed by the European Community Fund FEDER through COMPETE Programa Operacional Factores de Competitividade (POFC). Foundation for Science and Technology (FCT), Lisbon, through project PEstC/CTM/LA0025/2011.

\section{REFERENCES}

[1] As Gerações mais Idosas, Série de Estudos, n 83, INE, Lisboa, 1999.

[2] A. Alvarenga, "Os Envelhecimentos da População e as suas Consequências na Zona Euro 11", Informação Internacional, vol. 2, Departamento de Perspetiva e Planeamento, Ministério da Educação, Lisboa, 2000.

[3] F. Sousa, L. Viola, L. Ferreira, G. Trevisan, D. Cunha, J. Alves and R. Simoes, "An ecosystem of products and systems for ambient intelligence the AAL4ALL users perspective"; in proceeding of 9th International Conference on Wearable Micro and Nano Technologies for Personalized Health, Porto, Portugal, June 2012.

[4] L. A. Rolo, "Sobrecarga e Satisfação com a Vida: a percea̧ão dos cuidadores informais de idosos". Universidade de Aveiro Secção Autónoma de Ciências da Saúde, 2009

[5] E. Sales (2003). "Family burden and quality of life. Quality of life research" 12. (suppl.1), 33-41, 2003

[6] Carers UK, Care and technology in the 21st century. London 2012

[7] J. Barroso, R. Cabero Y Romero, "Las Personnas Mayores E Las Nuevas Tecnologias", Una Accin En La Sociedad De La Información, Innovación Educativa 12, 319-337, 2002

[8] J. Garcia Mínguez, A. Y. Sanchez, "Un Modelo De Educación En Los Mayores: La Interactividad”. Madrid Dynkinson, 1999.
[9] T.N. Robinson, K. Patrick, T.R.Eng D. Gustafson "An evidence-based approach to interactive health communication: A challenge to medicine in the information age". JAMA 1998; 280(14):1264-1269

[10] L.K. Wright, G. Bennet, L. Gramling. "Telecommunication interventions for caregivers of elders with dementia. Advances in Nursing Science" 1998; 20(3):76-88

[11] R. Schulz, S. Beach, "Caregiving as a risk factor for mortality: The caregiver health effects study". Journal of the American Medical Association 1999; 282:2215-2219

[12] D. Biegel, E. Sales, R. Schulz, "Family caregiving in chronic illness: Heart disease, cancer,stroke, Alzheimer's Disease, and chronic mental illness" Newbury Park: Sage Publications; 1993 